Pacific Journal of Mathematic 


\title{
UNIQUE FACTORIZATION OF RATIONAL HOMOTOPY TYPES
}

\author{
RICHARD BODY AND ROY DOUGLAS
}

\begin{abstract}
A nontrivial, simply connected, rational homotopy type is called irreducible, unless it is the product of two nontrivial rational homotopy types. In this paper previous results are extended by proving that every finitary, simply connected rational homotopy type having positive weights is representable as the product of a unique set of irreducible types. On the way to this unique factorization result, it is proven that (in the rational homotopy category) retracts of positive weight types again have positive weights.
\end{abstract}

In contrast to the (above) uniqueness result for the rational homotopy category, unique factorization fails simultaneously (i.e., with a single example) in three finer geometric contexts: the differentiable, topological and (integral) homotopy categories. This well-known noncancellation example is discussed in the introduction of [1].

The results in this paper were announced in [9].

Let $\mathscr{P} \mathscr{F}$ denote the set of all finitary, simply connected rational homotopy types having positive weights. The term "finitary" means that either the rational homotopy or the rational cohomology is a finite dimensional vector space over $Q$, the field of rational numbers. The term "positive weight" is defined in the next section. $\mathscr{P} \mathscr{F}$ contains the types of many interesting spaces (cf. [3], [6], [10], [12], [14]), including all simply connected, formal, finitary types. In fact, it is not an entirely trivial task to construct an example of a space which does not have positive weights. One of the earliest and simplest examples of such a space was constructed by Mimura and Toda [13] as the cofibre of a certain element of $\pi_{11}\left(S^{3} \vee C P(2)\right)$.

Throughout this paper, a rational homotopy type will be identified by its "minimal algebra" (or "minimal model" $d: M \rightarrow M$ denoted simply as $M$ ), where $M$ is a free, simply-connected, graded-commutative $Q$-algebra and $d$ is a decomposable, graded-derivation of degree 1 , which is a differential on $M$. A self-contained introduction to this view of rational homotopy theory may be found in [5], [7] or [11]; a demonstration of the usefulness of this view may be found in [15].

1. Rational homotopy types having positive weights. This section describes several equivalent characterizations of the set of 
rational homotopy types having "positive weights".

Definition. If each degree component of a minimal algebra $M=\bigoplus_{k \geqq 0} M^{k}$ decomposes as a direct sum,

$$
M^{k}=\bigoplus_{n \in Z}{ }_{n} M^{k}, \quad k \geqq 0 \quad(Z=\text { integers }),
$$

such that

(i) $d\left({ }_{n} M^{k}\right) \subset{ }_{n} M^{k+1}$ and

(ii) ${ }_{n} M^{k} \wedge{ }_{m} M^{l} \subset_{m+n} M^{k+l}$,

then this will be referred to as a weight decomposition, and the nonzero elements of ${ }_{n} M=\bigoplus_{k \geq 0} M^{k}$ will be said to have weight $n$.

The trivial weight decomposition is obtained by setting ${ }_{0} M^{k}=M^{k}$ and ${ }_{n} M^{k}=0$, for all $n \in Z, n \neq 0$, and $k \geqq 0$.

In [1] (Lemma 6, (4)) we observe that formal rational homotopy types possess a weight decomposition satisfying

$$
{ }_{n} M^{k}=0 \text {, if } n<k \text {. }
$$

Therefore, the augmentation ideal $M^{+}=\bigoplus_{k \geqq 1} M^{k}$ may be assigned strictly positive weights whenever $M$ is formal.

DEFINITION OF POSITIVE WEIGHT. A rational homotopy type $M$ has positive weights, if $M$ possesses a weight decomposition satisfying

$$
{ }_{n} M^{k}=0 \text {, if } k \geqq 1 \text { and } n \leqq 0 .
$$

(Of course, $M^{0}={ }_{0} M^{0} \simeq Q$.)

A useful property of positive weight rational homotopy types may be illustrated by considering an example of a rational homotopy type in $\mathscr{P} \mathscr{F}$ which is not formal. Let $M$ be the free, gradedcommutative $Q$-algebra generated by $\{a, b, x, y\}$, where degree $(a)=$ degree $(b)=2$, and degree $(x)=$ degree $(y)=5$; let $d: M \rightarrow M$ be the degree 1, graded derivation defined by $d(a)=d(b)=0, d(x)=a^{2} b$, and $d(y)=a b^{2} . \quad M$ is not formal, as there are nonvanishing Massey products in $H^{*}(M)$ (e.g., $x b-y a$ is a cocycle representing such a Massey product). However, $M$ is in $\mathscr{P} \mathscr{F}$; we can assign weight 2 to both $a$ and $b$; from this it follows that both $x$ and $y$ have weight 6 , thereby generating a positive weight decomposition of $M$. Let $\alpha_{t}: M \rightarrow M$ be defined by $\alpha_{t}(z)=t^{n} \cdot z$, where $n=$ weight $(z)$ and $t$ is any nonzero rational number. Then $\alpha: Q^{*} \rightarrow \operatorname{Aut}(M)\left(t \rightarrow \alpha_{t}\right)$ is a one-parameter (multiplicative) subgroup of the algebraic group Aut $(M)$, and $\alpha$ converges in the Zariski topology on End $(M)$ to the basepoint endomorphism (cf. [1]). The following theorem shows that this example is typical for positive weight types. 
THeOREM 1. If $X$ is a space with rational homotopy type $M$, then the following are equivalent.

(A) $M$ has positive weights.

(B) There exists a one-parameter subgroup $\lambda: Q^{*} \rightarrow \operatorname{Aut}(M)$ (in the algebraic group of automorphisms of $M$ ) which converges to the basepoint endomorphism $O_{M}$ in End $(M)$ (with the Zariski topology), (cf. [1]).

(C) The Zariski closure of Aut $(M)$ in End $(M)$ contains the basepoint endomorphism.

Of course, strictly speaking, End $(M)$ (resp., Aut $(M)$ ) is the set of Q-rational points in a variety (resp., linear algebraic group), which is defined over $Q$. By an abuse of language, we will speak only of the $Q$-rational points; however, such considerations become more subtle in another context ([2], [8]) and there the discussion is not abbreviated by this abuse.

The proof that (A) implies (B) follows easily by the construction of a one-parameter subgroup using the positive weight decomposition, precisely as illustrated in the example preceding Theorem 1.

Of course, (B) implies (C) is an obvious remark.

To see that (C) implies (B) requires the following observations.

(1) Without loss of generality, assume $O_{M}$ is in the closure of the connected component of the unit element $1_{M} \in \operatorname{Aut}(M)$.

(2) $O_{M}$ is in the closure of a connected algebraic group implies $O_{M}$ is in the closure of a maximal $Q$-split torus.

(3) Analysis of the action of the torus on its closure, where $O_{M}$ is a fixed point, implies (B).

Finally, (B) implies (A), because we can diagonalize any (nonunit) element of the one-parameter subgroup of automorphisms and use its eigenspaces to give the weight decomposition required in (A).

This completes our outline of the proof of Theorem 1 .

REMARK. If $X$ is a finitary nilpotent $C W$ complex, then $X$ "telescopes" to a $p$-localization (for at least one prime, and therefore all primes, $p$ ), if and only if $M$ has positive weights, (cf. [3]). If $X$ is a simply-connected, finite complex, then $X$ is $p$-universal (for at least one prime, $p$, and therefore all primes, $p$ ), if and only if $M$ has positive weights, (cf. [3] and [13]).

In the remainder of this paper we will use only conditions (B) and $(\mathrm{C})$. For the details of the proofs for Theorem 1 and the above remark, see [3].

Recall that the categorical coproduct is the tensor product of minimal algebras $\otimes$, which may be viewed as the product space construction. Thus, nontrivial minimal algebras are called irreduci- 
ble, if they are not the tensor product of two nontrivial minimal algebras.

Proposition 2. Suppose $M$ and $N$ are minimal algebras. Then $M \otimes N$ has positive weights if and only if both $M$ and $N$ have positive weights.

Proof. If $M$ and $N$ have positive weights, then it is easy to show that $M \otimes N$ has positive weights (using condition (B) in Theorem 1).

Conversely, suppose $M \otimes N$ satisfies condition (B); it suffices to show that $M$ satisfies condition (C). In fact, this argument is valid for arbitrary "retracts", and the following lemma completes the proof of Proposition 2.

Lemma 3. Suppose $p: M \rightarrow N$ and $i: N \rightarrow M$ are D.G.A. morphisms of minimal models such that $(p \circ i) \in \operatorname{Aut}(N)$. If $M$ has positive weights, then $N$ must also have positive weights.

Proof of lemma. Without loss of generality, assume $p \circ i=1_{N}$. Let $\Psi:$ End $(M) \rightarrow$ End $(N)$ be defined by $\Psi(f)=p \circ f \circ i$. $\Psi$ is continuous and by (B), there exists a 1-parameter subgroup $\lambda: Q^{*} \rightarrow$ Aut $(M)$ which converges to $O_{M} \in \operatorname{End}(M)$. Let $L=\lambda\left(Q^{*}\right) \subset \operatorname{Aut}(M)$ and let $\bar{L}$ be the Zariski closure of $L$ in End $(M)$. Since $L$ is a connected algebraic subgroup of $\operatorname{Aut}(M), L$ is an irreducible variety (not the union of two proper closed subsets). An elementary argument shows that $\bar{L}$ is a irreducible subvariety of $\operatorname{End}(M)$ and $S=$ $\Psi(\bar{L})$ is an irreducible subspace of End $(N)$.

$$
1_{N} \in \operatorname{Aut}(N) \cap S=D \neq \varnothing .
$$

Thus, $D$ is a nonempty, open subset of $S$. It is easy to show that $S \subset \bar{D}$, because $S$ is irreducible ( $\bar{D}$ is the Zariski closure of $D$ in End $(N))$. Finally, we observe that

$$
O_{N} \in \Psi(\bar{L})=S \subset \bar{D} \subset \overline{\operatorname{Aut}(N)} \subset \operatorname{End}(N),
$$

which gives condition (C) for $N$.

2. Unique factorizaton for $\mathscr{P} \mathscr{F}$. Consider the minimal subalgebra, $M(k) \subset M$ generated by the generators of $M$ of degree $\leqq k$. $M(k)$ is the minimal algebra corresponding to the $k$ th Postnikov section of $M$.

Of course, if $M(k)$ is irreducible for some $k$ and $H^{p}(M)=0$ for $p>k$, then $M(l), l \geqq k$, and $M$ are irreducible. The following 
lemma supplies a partial converse.

Lemma 4. If $M$ is irreducible and $H^{i}(M)=0$ for $i>k$, then $M(2 k-1)$ is irreducible.

Proof. Suppose $M(2 k-1)$ is not irreducible. Then $M(2 k-1) \simeq$ $M^{\prime} \otimes M^{\prime \prime}$ (with $M^{\prime}$ and $M^{\prime \prime}$ nontrivial) and $H^{j}\left(M^{\prime}\right)=0=H^{j}\left(M^{\prime \prime}\right)$ for $k<j<2 k+1$. Observe that if $N$ and $N^{\prime}$ are minimal algebras, with $N(m) \simeq N^{\prime}(m)$, and $H^{j}(N)=H^{j}\left(N^{\prime}\right)=0$ for $j>m$, then $N \simeq N^{\prime}$. Thus, there exist unique minimal algebras $N$ and $L$ with $N(2 k-1) \simeq M^{\prime}, L(2 k-1) \simeq M^{\prime \prime}$, and $H^{j}(N)=H^{j}(L)=0$ for $j>k$.

The inclusion of $N(2 k-1)$ into $M$ extends to a D.G.A. map $N \rightarrow M$. Similarly, the inclusion of $L(2 k-1)$ into $M$ extends to a D.G.A. map $L \rightarrow M$, and this gives a D.G.A. map $N \otimes L \rightarrow M$ which induces an epimorphism on cohomology.

Because $H^{*}(N \otimes L) \simeq H^{*}(M)$, the induced epimorphism $H^{*}(N \otimes$ $L) \rightarrow H^{*}(M)$ is an isomorphism (each gradation is finite dimensional), and as such is induced by an isomorphism of minimal algebras, $N \otimes L \rightarrow M$ ([7], Corollary 2.7). Thus, $M$ is not irreducible.

REMARK. Lemma 4 can also be seen as an elementary fact about Postnikov towers. The one-point-union of two $k$-spheres $S^{k} \vee S^{k}$ shows that Lemma 4 cannot be strengthened in the obvious way.

The main result in this paper can be stated precisely as:

THEOREM 5. Every minimal algebra in $\mathscr{P} \mathscr{F}$ is the tensor product of a unique set of irreducible minimal algebras.

Proof. The proof of Proposition 7 in [1], taken together with Proposition 2 (above), actually proves that finitely generated minimal algebras having positive weights are "flexible" (cf. [1]). Then an appeal to Theorem 2 in [1] completes this proof for the finitely generated minimal algebras of $\mathscr{P} \mathscr{F}$.

Now suppose $M$ has positive weights and finite dimensional cohomology; say $H^{i}(H)=0$, for $i>k$. It is easy to show that Postnikov sections of $M$ also have positive weights (by using condition (B) of Theorem 1). Thus, $M(l)$ has positive weights, for each $l>0$.

If $M \simeq \bigotimes_{j=1}^{m} M_{j}$ and each $M_{j}$ is irreducible, then for each $l>0$

$$
M(l) \simeq \bigotimes_{j=1}^{m} M_{j}(l),
$$

and each of the $M_{j}(l)$ have positive weights (by Proposition 2). 
Moreover, each $M_{j}(l)$ is irreducible for $l \geqq 2 k-1$ (by Lemma 4).

Unique factorization for $M(l)$ follows from the first part of the proof of Theorem 5. However, by the above observations, the set of irreducible tensor product factors of $M$ and $M(l)$ correspond bijectively, if $l \geqq 2 k-1$. Thus, the unique factorization of $M(l)$, for each (or any) $l \geqq 2 k-1$, implies unique factorization for $M$.

\section{REFERENCES}

1. R. Body and R. Douglas, Rational homotopy and unique factorization, Pacific Journal Math., 75 (1978), 331-338.

2. - Tensor products of graded algebras and unique factorization, Amer. J. Math., 101 (1979), 909-914.

3. R. Body and D. Sullivan, Homotopy types which telescope (or "Zariski dynamics of a homotopy type"), preprint, U.C.S.D., La Jolla, California (to be submitted to Topology).

4. A. Borel, Linear Algebraic Groups, W. A. Benjamin, New York, 1969.

5. A. K. Bousfield and V.K.A.M. Gugenheim, On PL de Rham theory and rational homotopy type, Memoirs of Amer. Math. Soc., 8, No. 179 (1976).

6. P. Deligne, P. Griffiths, J. Morgan and D. Sullivan, Real homotopy theory of Kähler manifolds, Inventiones Math., 29 (1975), 245-274.

7. A. Deschner, Sullivan's theory of minimal models, thesis (and preprint), University of British Columbia, 1976.

8. R. Douglas, The uniqueness of coproduct decompositions for algebras over a field, Proceedings of Algebraic Topology Conference, Vancouver (1977), Lecture Notes in Mathematics, Vol. 673, Springer-Verlag.

9. Unique factorization for rational homotopy types and graded algebras, Abstract for special session on algebraic topology, Notices of Amer. Math. Soc., 24 No. 5, p. A-499 (1977), [Abstract*747-55-12].

10. H. Glover, W. Homer and G. Mislin, Immersions in manifolds of positive weights, Proceedings of Algebraic Topology Conference, Vancouver (1977), Lecture Notes in Mathematics, Vol. 673, Springer-Verlag.

11. S. Halperin, Lectures on Minimal Models, notes from University of Lille I, 1977.

12. M. Mimura, G. Nishida and H. Toda, Localization of CW-complexes and its applications, J. Math. Soc. Japan, 23 (1971), 593-624.

13. M. Mimura and H. Toda, On p-equivalences and p-universal spaces, Comment. Math. Helv., 46 (1971), 87-97.

14. J. Morgan, Homotopy theory of smooth algebraic varieties, I.H.E.S., publications, No. $48,1978$.

15. D. Sullivan, Infinitesimal computations in topology, I.H.E.S. Publications, No. 47, 1977.

Received February 6, 1979. Second named author's research partly supported by N.S.E.R.C. of Canada.

University of Western Ontario

AND

University of British Columbia

Vancouver, B.C., Canada V6T 1 W5 


\section{PACIFIC JOURNAL OF MATHEMATICS}

\section{EDITORS}

DONALD BABBITT (Managing Editor)

University of Galifornia

Los Angeles, California 90024

HUGO RossI

University of Utah

Salt Lake City, UT 84112

C. C. MOORE AND ANDREW OGG

University of California

Berkeley, CA 94720
J. DUGUNDJI

Department of Mathematics University of Southern California Los Angeles, California 90007

R. FINN AND J. MILGRAM Stanford University

Stanford, California 94305

ASSOCIATE EDITORS

E. F. BECKENBACH

B. H. NeumanN

F. WOLF

K. YosHidA

\section{SUPPORTING INSTITUTIONS}

UNIVERSITY OF BRITISH COLUMBIA UNIVERSITY OF SOUTHERN CALIFONIA CALIFORNIA INSTITUTE OF TECHNOLOGY UNIVERSITY OF CALIFORNIA MONTANA STATE UNIVERSITY STANFORD UNIVERSITY UNIVERSITY OF HAWAII UNIVERSITY OF NEVADA, RENO UNIVERSITY OF TOKYO U'NIVERSITY OF UTAH NEW MEXICO STATE UNIVERSITY WASHINGTON STATE UNIVERSITY OREGON STATE UNIVERSITY UNIVERSITY OF OREGON UNIVERSITY OF WASHINGTON 


\section{Pacific Journal of Mathematics}

\section{Vol. 90, No. $1 \quad$ September, 1980}

Shashi Prabha Arya and M. K. Singal, On the locally countable sum

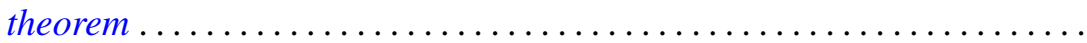

John Theodore Baldwin and David William Kueker, Ramsey quantifiers and

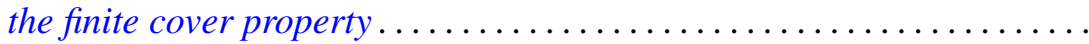

Richard Body and Roy Rene Douglas, Unique factorization of rational

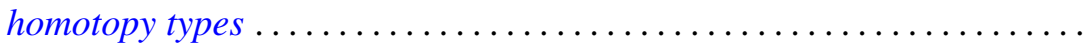

Ethan Bolker and Ben G. Roth, When is a bipartite graph a rigid

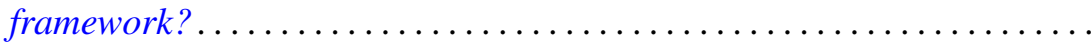

Alicia B. Winslow, Continua in the Stone-Čech remainder of $R^{2} \ldots \ldots \ldots$

Richard D. Carmichael and Elmer Kinji Hayashi, Analytic functions in tubes which are representable by Fourier-Laplace integrals ..............

Stephen D. Cohen, The Galois group of a polynomial with two indeterminate coefficients ..............................

Russell Allan Johnson, Strong liftings commuting with minimal distal

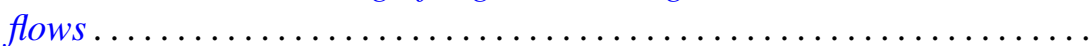

Elgin Harold Johnston, The boundary modulus of continuity of harmonic

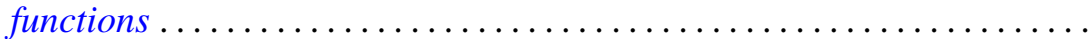

Akio Kawauchi and Takao Matumoto, An estimate of infinite cyclic

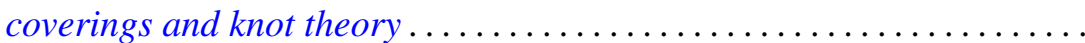

Keith Milo Kendig, Moiré phenomena in algebraic geometry: rational alternations in $\mathbf{R}^{2}$...

Roger T. Lewis and Lynne C. Wright, Comparison and oscillation criteria for selfadjoint vector-matrix differential equations .

Teck Cheong Lim, Asymptotic centers and nonexpansive mappings in conjugate Banach spaces .......................

David John Lutzer and Robert Allen McCoy, Category in function spaces.

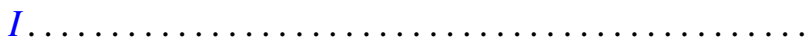

Richard A. Mollin, Induced p-elements in the Schur group ...

Jonathan Simon, Wirtinger approximations and the knot groups of $F^{n}$ in $S^{n+2}$

Robert L. Snider, The zero divisor conjecture for some solvable groups...

H. M. (Hari Mohan) Srivastava, A note on the Konhauser sets of biorthogonal polynomials suggested by the Laguerre polynomials...

Nicholas Th. Varopoulos, A probabilistic proof of the Garnett-Jones theorem on BMO.

Frank Arvey Wattenberg, $[0, \infty]$-valued, translation invariant measures on $N$ and the Dedekind completion of ${ }^{*} R \ldots \ldots \ldots \ldots . .$. 